\title{
Young and old massive star clusters and their stellar populations - Theoretical challenges for the next decade
}

\author{
Corinne Charbonnel ${ }^{1,2}$ \\ ${ }^{1}$ Department of Astronomy, University of Geneva \\ 1290 Versoix - Switzerland \\ email: Corinne.Charbonnel@unige.ch \\ ${ }^{2}$ IRAP CNRS UMR 5277, Université de Toulouse III \\ 31400 Toulouse, France
}

\begin{abstract}
Several models presented in the literature compete to explain the origin of multiple stellar populations in globular clusters (GC), but they all fail to reproduce the large variety of present-day characteristics of these systems. In parallel, independent clues on GC early evolution may be derived from observations of young massive clusters (YMC) in the Local Group. But are these two populations of clusters related? And can we reconcile the informations and data concerning GCs and YMCs? Here we summarize some open questions on the nucleosynthetic origin of multiple stellar populations in GCs, on the actual evolution and characteristics of GC low-mass stars, and on early gas expulsion from massive clusters. We propose theoretical paths to be explored in the near future.
\end{abstract}

Keywords. globular clusters: general

\section{Introduction}

Massive clusters of stars (MSC), young and old, are magnificent astronomical objects, and keys to a broad variety of astronomical and cosmological questions. They are fundamental benchmark for stellar evolution theory, key sites for the formation of the most massive stars, and candidate host of gamma ray bursts. They are unique witnesses and clocks of the formation and assembly of galaxies and of their substructures, from the early to the present-day Universe. They play a key role in hierarchical cosmology and potentially also in the reionization of the Universe. However, their formation, evolution, and survival are far from being understood. Comparative studies of MSCs, young and old, in the Milky Way, Local Group, starburst and interacting galaxies are now possible thanks to exquisite observations performed e.g. with HST, VLT, Herschel, and ALMA. Those try to bring gap(s) and make links between young and old MSCs, revealing precious informations and bringing extraordinary surprises. All these scientific issues were at the heart of the scientific rationale we proposed for IAUS316, and they have been extensively addressed during that international conference at the IAU General Assembly in Honolulu. We refer to the reviews and contributions presented in this Volume for the most recent significant results and literature references.

Many of the conversations at IAUS316 were dominated by the debate on the explanation of multiple stellar populations (MSPs) in globular clusters (GCs). In this paper we summarize a couple of the many theoretical challenges that have been raised by the discovery of these MSPs $(\S 2)$, in particular the nucleosynthetic origin of the abundance 
anomalies in GCs (§3), the actual evolution and fate of GC low-mass stars (§4), and the mass budget problem under new insight from extragalactic young MSCs ( 55 ).

\section{Multiple stellar populations (MSP) in globular clusters - Open questions}

One of the major breakthroughs of the past decade in the domain concerns those MSCs we thought we knew the best, namely the globular clusters (GC). It was long taken for granted that individual GCs were the perfect archetype of a single stellar population, and that they had undergone no internal chemical evolution (except for the most massive ones like Omega Cen, which is not even considered as a GC but rather as the remnant of a small galaxy), nor any drastic modification of e.g. their mass along their life. This classical view is at odds with the new paradigm imposed by modern spectroscopic and photometric observations. Indeed we know now that these old systems actually host MSPs. Those exhibit very peculiar chemical properties and leave unexpected imprints in the clusters color-magnitude diagrams that have never been discovered yet in any other stellar population, nor in the field, nor in open clusters (e.g. the reviews by Bragaglia, Marino, and Piotto, this Volume, and references therein).

Extensive literature is devoted to possible explanations to the ubiquitous presence of MSPs in GCs, as well as their unique spectroscopic and photometric complexity. However, none of the proposed scenarii that call for chemical self-enrichment of GCs in their enfancy is able to reconcile all the available data (e.g. Bastian, this Volume). Therefore, we are left with very basic, but crucial questions: Which type of short-lived massive stars did pollute the intracluster material with only hydrogen-burning products during the early GC infancy? How did the multiple populations of low-mass stars that are still alive today form in each individual GC, how did they inherite their chemical peculiarities, and what are the consequences for their evolution and fate? Why are these MSPs not observed in other types of star clusters, like open clusters? Does this reflect very specific star formation mechanisms in the early universe? Or will we also find similar MSPs in young massive star clusters formed in present-day conditions once we will have the suited observational tools? What was the original mass of GCs, and their contribution to galactic haloes? Old GCs consist today of old low-mass stars and little or no gas. However, there must have been a time when GCs formed as gas-rich objects, hosting numerous massive, short-lived stars. What has been the chemical and dynamical impact of these massive stars on their host clusters? How did the feedback with the intra-cluster medium (ICM) impact the formation and evolution of stars on various temporal and spatial scales, as well as the dynamics of the entire clusters? Did they contribute to gas ejection eventually associated to a significant loss of stars without disrupting the cluster by a drastic change of potential well?

All these questions constitute for sure the most important theoretical challenges in the domain for the next (couple of?) decade(s). Here we point out some of the most critical issues.

\section{Have we identified the nucleosynthetic origin of the chemical properties of MSPs in GCs?}

About two thirds of GC stars exhibit chemical properties that reflect the signatures of hydrogen-burning at high temperature through $\mathrm{CNO}$-cycle and $\mathrm{NeNa}$ and $\mathrm{MgAl}$ chains 
(e.g. Prantzos et al. 2007). This proportion of so-called 2d population stars (2P $\dagger$ ) is relatively constant from cluster to cluster (e.g. Prantzos \& Charbonnel 2006, Carretta 2010 ); it is almost independent of the stellar evolution stage (main sequence and red giant branch), althought it slightly differs from cluster to cluster among AGB stars (e.g. Wang et al., this Volume). This is currenty interpreted as the result of self-enrichment of GCs in their early infancy, with $2 \mathrm{P}$ stars having formed out of the H-ashes of massive $1 \mathrm{P}$ stars mixed with original proto-cluster gas. The most-commonly invoked polluters are the socalled Fast Rotating Massive Stars (FRMS, $\geqslant 20 \mathrm{M}_{\odot}$; Decressin et al. 2007a, Decressin et al. 2007b, Krause et al. 2013), and massive AGB stars ( 6 - $6.5 \mathrm{M}_{\odot}$; e.g. Ventura et al. 2001, Ventura et al. 2013, D'Ercole et al. 2010). Variants and combinations of FRMS and AGB have been proposed (e.g. Sills \& Glebbeek 2010, Bastian et al. 2013), as well as intermediate-mass binaries $\left(10-20 \mathrm{M}_{\odot}\right.$; De Mink et al. 2009) and supermassive stars $\left(\sim 10^{4} \mathrm{M}_{\odot}\right.$ stars; Denissenkov \& Hartwick 2014).

However, all these propositions face difficulties to explain simultaneously all the observed abundance patterns. For example, all the AGB models computed by independent groups do predict AGB yields at odds with the observed O-Na anticorrelation (e.g. Forestini \& Charbonnel 1997, Denissenkov \& Herwig 2003, Karakas \& Lattanzio 2007, Ventura et al. 2013). Additionally, state-of-the-art rotating models of AGB stars predict that those stars should also eject helium-burning products, which is sustained by a wide variety of galactic observations (e.g. Maeder \& Meynet 2006, Chiappini et al. 2008), but which is at odds with the constancy of $\mathrm{C}+\mathrm{N}+\mathrm{O}$ observed among GC lowmass stars (Decressin et al. 2009). Attempts to "save" (quoting the authors) the AGB scenario call for adhoc dilution of AGB ejecta with galactic gas of the exact same composition as that of the original proto-cluster that must be re-accreted by all GCs about 50 to 100 Myrs after the expulsion of the original proto-cluster gas and of the supernovae (SNe) ejecta material (D'Ercole et al. 2012). Some call for a major revision of the cross-section of a critical reaction rate destroying sodium as an alternative option (Renzini et al. 2015).

In the case of FRMS being the polluters, a revision of the cross section of the reaction ${ }^{24} \mathrm{Mg}(\mathrm{p}, \gamma)$ is advocated for in order to reproduce the amplitude of the observed $\mathrm{Mg}-\mathrm{Al}$ anticorrelation among GC stars (Decressin et al. 2007a). In addition, the different mechanisms that may lead to equatorial mass ejections of H-burning ashes when massive stars reach break-up have not yet been fully investigated, so that the actual mass of the H-ashes that can be released by FRMS, especially very early on the main sequence when the Na-rich material is not yet strongly enriched in helium, might well be underestimated. Intermediate-mass binaries being the polluters also suffers some caveats (D'Antona et al. 2012), one being their internal temperature being too cool to explain the observed nucleosynthetic patterns (Prantzos et al. 2007).

Importantly, all the current polluter models face difficulties to account for the helium differences between $1 \mathrm{P}$ and $2 \mathrm{P}$ stars that have been derived from analyses of the spreads in the main-sequence colors in ultraviolet and optical filters for a handful of GCs (Bastian et al. 2015, and references therein). In this respect, supermassive stars might be promising from the nucleosynthetic point of view. However, this requires the verification of speculations on how the required mass-loss might be caused by the super-Eddington radiation continuum-driven stellar wind or by the diffusive mode of the Jeans instability. Moreover, no self-consistent scenario linking all the chemical and dynamical constraints has been proposed so far for these hypothetic stars.

$\dagger$ Compare with the 1st population, 1P, that shows chemical composition similar to that of halo stars. 
Therefore, the exact nature of the first population GCs polluters is far from being definitively ascertained. New stellar models including internal magneto-hydrodynamical processes (rotation, mass loss, magnetic fields, hydrodynamical instabilities) over the whole initial stellar mass range as well as the whole metallicity range covered by GCs are definitively needed to probe the nucleosynthetic origin of GC stellar properties.

We can therefore conclude that GCs will be for long extraordinary exploration territories for stellar physics.

\section{How does the peculiar initial composition of GC low-mass stars impact their evolution?}

Old GCs contain today only low-mass stars (below $\sim 0.85 \mathrm{M}_{\odot}$ ) and the dark remnants of the upper end of the initial stellar mass function (IMF). Despite the many differences between the scenarios that have been developed to explain the abundance properties of GCs $(\S 3)$, it is well accepted that $2 \mathrm{P}$ GC stars have started their life with a higher helium content than their $1 \mathrm{P}$ counterparts. This is confirmed by direct spectroscopic measurements of non-local thermodynamic equilibrium $\mathrm{He}$ abundances for a subset of blue horizontal branch stars in the GC NGC 2808 (Marino et al. 2014). It is therefore mandatory to account for their non-standard helium content on the evolution of low-mass stars to account for all the spectroscopic and photometric observational constraints, as the helium content of stars does definitively impact their evolution, lifetime, and fate.

Predictions for the extent of helium enrichment along the sodium distribution in $2 \mathrm{P}$ stars strongly depend on the nature of the invoked $1 \mathrm{P}$ polluters. In the AGB scenario, all $2 \mathrm{P}$ stars (i.e., spanning a large range of $\mathrm{Na}$ abundances) are expected to be born with very similar helium contents (maximum of $0.36-0.38$ in mass fraction, e.g. Doherty et al. 2014, if no dilution with the ISM matter is taken into account, to be compared with $\sim 0.248$ for $1 \mathrm{P}$ stars $)$. In contrast, the FRMS scenario predicts broad and correlated spreads of both helium and sodium in the initial mixture that formed $2 \mathrm{P}$ stars, with initial helium mass fraction ranging between 0.248 (as for the $1 \mathrm{P}$ ) and 0.8 in the most extreme 2P stars (Decressin et al. 2007b). This has noticeable consequences on the evolution and fate of GC 2P stars (details in Chantereau et al. 2015; see also Chantereau et al., this Volume). Such initial abundances provide e.g. a straightforward explanation to the lack of sodium-rich 2P AGB stars in GCs like NGC 6752 (Charbonnel et al. 2013; observations by Campbell et al. 2013) due to the impact of the initial helium content on the stellar lifetime and evolution path in the Hertzsprung-Russel diagram. They also account for the finding of different sodium spreads that are observed along the AGB in the Galactic globular clusters of different ages and $[\mathrm{Fe} / \mathrm{H}]$ values (Charbonnel \& Chantereau 2015). Simultaneously, the FRMS scenario in its current form predicts that at a typical GC age of 13.4 Gyr, $95 \%$ of the low-mass stars lying two magnitudes below the turn-off should be born with a helium mass fraction $\mathrm{Y}_{\text {ini }}$ between 0.248 and 0.4 , and only $5 \%$ with $\mathrm{Y}_{\text {ini }}$ higher than 0.4 (Chantereau et al. 2016; Chantereau et al., this Volume); no star born with $\mathrm{Y}_{\text {ini }}$ higher than 0.4 are expected to lie today on the horizontal branch, in agreement with current interpretations of GC horizontal branch morphologies in connection with the so-called second parameter problem (e.g. D'Antona \& Caloi 2004, Gratton et al. 2010, Dotter et al. 2010).

Although the initial helium distribution among $2 \mathrm{P}$ stars is not well known yet, all the polluter models presently fail to account for the extremely low helium enrichment deduced from main sequence color spreads in some GCs (e.g. NGC 6752, Milone et al. 2013). As of today, the maximum helium spread for a GC was derived in NGC 2808 (Milone et al. 
12b), and large differences in He spreads have been found between clusters. This clearly poses a problem to all the polluters proposed in the literature, at least within the current frameworks, and it requires large degrees of stochasticity in the polluter yields (Bastian et al. 2015).

One has to note however that a direct and reliable comparison between theoretical predictions and observed GC CMDs is still hampered for several reasons. Going from the theoretical to the observational plane requires indeed the use of model atmospheres and temperature-color transformations suited for the proper helium range and associated peculiar composition of $2 \mathrm{P}$ stars. This has already been done up to a certain extent (Sbordone et al. 2011, Milone et al. 2013, Cassisi et al. 2013), but not for large helium abundance spread, as such tools are not yet available. In addition, from the stellar evolution point of view we need to go one step further by computing models of $1 \mathrm{P}$ and $2 \mathrm{P}$ long-lived low-mass stars taking into account the effects of the atomic diffusion, rotation, and mixing, which are expected to have a non negligible impact on stellar evolution and lifetimes, and on the position of stars within the HRD. Work is in progress in these complementary directions.

\section{Is massive gas ejection the solution to the mass budget problem for GCs? Insight from extragalactic YMCs}

One critical issue to account for MSPs in GCs is that, when one assumes a classical IMF for the potential stellar polluters, the ratio between $2 \mathrm{P}$ and $1 \mathrm{P}$ stars is predicted to be 10 to $90 \%$; this is at odds with the observed ratio $70 \%-30 \%$ quoted above (Prantzos \& Charbonnel 2006). Variants and combinations of the different potential polluters have been proposed to alleviate the mass budget issue, but this actually strengthens the nucleosynthetic issues that we briefly recalled in $\S 3$. To enhance the $2 \mathrm{P}$ to $1 \mathrm{P}$ ratio, one can alternatively call for a flat IMF for the polluters (Smith \& Norris 1982, D'Antona \& Caloi 2004, Prantzos \& Charbonnel 2006, Schaerer \& Charbonnel 2011), or for a 1P composed of massive stars only giving birth to all the low-mass stars we observe today in GCs (Charbonnel et al. 2014). While these options might open extremely interesting avenues, their many implications have not yet been investigated in detail.

As of today, the most commonly invoked solution to the mass budget issue calls for fast and drastic gas expulsion that would have lead to the loss of the vast majority of $1 \mathrm{P}$ low-mass stars while $2 \mathrm{P}$ stars formed in the center of GCs would have remained bound to the cluster (e.g. Prantzos \& Charbonnel 2006, Decressin et al. 2010, D'Ercole et al. 2010, Schaerer \& Charbonnel 2011, Krause et al. 2013, Khalaj \& Baumgardt 2015).

Models of gas expulsion considering superbubbles kinematics indicate however that stellar winds and supernovae explosions may not be sufficient to accomplish the task, except for the least massive and most extended clusters. Alternatively, coherent onset accretion onto $1 \mathrm{P}$ neutron stars and stellar black holes might do it for the whole range of GC compactness. However in that case, gas ejection would happen only after all $1 \mathrm{P}$ massive stars have turned into such dark remnants at the end of the SNe phase, i.e., typically after $\sim 35$ Myr after cluster formation (see Krause et al. 2012, Krause et al. 2013 for details).

This delay for gas expulsion is in tension with observations of young MCs (YMC) in the Local Group and in dwarf galaxies. Indeed, some of these objects with ages below $10 \mathrm{Myr}$, current masses between 8 and $50 \times 10^{5} \mathrm{M}_{\odot}$, and half-mass radii between 1.5 and $18 \mathrm{pc}$ (i.e., very similar to the expected properties of GC progenitors), appear to be gas free by an age of $\sim 3 \mathrm{Myr}$, and show no evidence for multiple epochs of star-formation (Bastian et al. 2014). In the case GC formation at high redshift happened the same way 
as YMC formation today, this implies that the dark remnant scenario for fast gas removal occurs too late to explain the disappearance of the gas at such an early stage. For very early gas expulsion to eventually work, one must then invoke either energy ejection by $10^{52}-10^{53}$ erg hypernovae, or a very high star formation efficiency (SFE from $50 \%$ in the most loose clusters, and up to more than $90 \%$ in the most compact ones), the efficiency of gas expulsion being extremely dependent on the cluster compactness and on local SFE (see details in Krause et al. 2016).

There is no definitive evidence yet that GC formation at high redshift was similar to YMC formation happening in the local and modern universe. However, if YMCs are the actual modern counterparts of GCs then gas and star expulsion is a serious issue for the current GC self-enrichment scenarii that make classical assumptions for the SFE and for the stellar IMF of the first and second stellar populations. Rather, gas could be cleared for very high SFE, but in that case the total stellar mass would not be changed significantly enough to affect the $1 \mathrm{P}$-to- $2 \mathrm{P}$ ratio as requested by the mass budget.

What can we do next? It clearly appears that the detection of the O-Na anticorrelation in YMCs could be a definitive clue to the "genetic" relationship between YMCs and GCs. On the theoretical side, analytical and semi-analytical modelling of the expansion and instabilities of self-gravitating superbubbles including for the first time relevant prescriptions from stellar models to describe stellar mass loss, winds, luminosity, and energy, have highlighted the very important role of stellar feedback on MSC dynamics. Refined and detailed 3D-hydrodynamical treatment of the subtle interactions between stellar ejecta (mass, energy, nucleosynthesis products), interstellar matter, and secondary star formation are definitively needed now to probe in detail all the aspects of the vast problem of the formation of MSPs in MSCs in the early phases of their evolution.

\section{Conclusions}

Old GCs contain today only old low-mass stars and the dark remnants of more massive, short-lived stars. Until about a decade ago, most of the studies related to these objects were implicitly considering that this had always been the case, and that the rest of the IMF had very little impact on the evolution of such clusters that managed to stay gravitationally bound for 10 to 13 billion years. The discovery of ubiquitous MSPs has revolutionized the field. It revealed that all GCs have actually undergone drastic chemical and dynamical processes in their early infancy. This has opened far-reaching questions for stellar and galactic physics, and for cosmology, that none of the scenarios proposed so far can solve. As we are trying to get complementary clues from YMCs in nearby and interacting galaxies, we have no definitive evidence that the formation mechanisms of these local objects happen through the same path than GC formation that occurred in the early Universe.

One of the conclusions of the discussions at IAUS 316 is that none of the presently circulating scenario can explain all the photometric and spectroscopic constraints. We may think we are at an impasse, but this is how science works at its best. We just need innovative thoughts and a different approach, out of the box. For sure, finding the answers will require exchange of ideas and close collaboration of astrophysicists with observational, theoretical, and numerical expertise in stellar evolution, interstellar matter magnetohydrodynamics, stellar dynamics, formation and evolution of galaxies, cosmology, multidimensional numerical simulations, N-body simulations, and multi-wavelength high-precision photometry, spectroscopy, and astrometry. Work is in progress and, hopefully, a robust and consensual explanation will be reached in the near future. 


\section{Acknowledgements}

I thank IAU for supporting the organization of IAUS 316 on Formation, Evolution, and Survival of Massive Star Clusters, as well as the SOC members. My warmest thoughts go to my fantastic co-chair, A.Nota, for the nice organizational and scientific adventures we shared. I acknowledge support from the Swiss National Science Foundation (FNS) for the project 200020-159543 "Multiple stellar populations in massive star clusters - Formation, evolution, dynamics, impact on galactic evolution" (PI CC). I thank the International Space Science Institute (ISSI, Bern, CH) for welcoming the activities of ISSI Team 271 "Massive star clusters across the Hubble Time (2013 - 2016, team leader CC), as well as my colleagues, M.Krause, N.Bastian and R.Diehl, co-authors of K15 paper, and F.Primas, W.Chantereau, and Y.Wang, co-authors of C14.

\section{References}

Bastian, N., et al., 2013, MNRAS, 436, 2398

Bastian, N., Hollyhead, K, \& Cabrera-Ziri, I., 2014, MNRAS, 445, 378

Bastian, N., Cabrera-Ziri, I., \& Salaris, M., 2015, MNRAS, 445, 378

Bastian, N., \& Lardo, C., 2015, MNRAS, 449, 3333

Bragaglia, A., et al. 2014, ApJ, 796, 68

Campbell, S. W., et al. 2013, Nature, 498, 198

Carretta, E.., et al. 2009, A\&A 505, 139

Carretta, E.., et al. 2010, A\&SA, 516, A55

Carretta, E. 2010, A\&A, 557, A128

Cassisi, S., Mucciarelli, A., Pientriferni, A., Salaris, M., \& Ferduson, J. 2013, A\&SA, 554, A19

Chantereau, W., Charbonnel, C., \& Decressin, T. 2015, A\&A, 578, A117

Chantereau, W., Charbonnel, C., \& Meynet, G. 2016, submitted to $A \& \mathcal{G} A$

Charbonnel, C. \& Krause, M., 2015, IAUS 317 "The General Assembly of the Galactic Halo", in press

Charbonnel, C. \& Lagarde, N. 2010, $A \& 3 A, 522$, A10

Charbonnel, C. \& Zahn, J. P. 2007, A\& A (Letter), 467, L15

Charbonnel, C., et al. 2013, A\&AA (Letter), 557, L17

Charbonnel, C., Chantereau, W., Krause, M., Primas, F., \& Wang, Y. 2014, A $\mathscr{J} A$ (Letter), 569, L6 (C14)

Charbonnel, C., \& Chantereau, W., 2015, A\&A, in press

Chiappini, C. 2008, A\&A, 479, L9

D'Antona, F. \& Caloi, V. 2004, ApJ, 611, 871

D'Antona, F., et al. 2012, A\&A A, 443, 3302

Decressin, T., et al. 2007a, A\& A, 464, 1029

Decressin, T., Charbonnel, C., \& Meynet, G, 2007b, $A \mathscr{E} A, 475,859$

Decressin, T., Charbonnel, C., et al. 2009, A\&BA, 505, 727

Decressin, T., Baumgardt, H., Charbonnel, C., \& Kroupa, P., 2010, A\&\&A, 516, A73

De Mink, S., et al. 2009, A\&GA (Letters), 507, L1

Denissenkov, P. A. \& Herwig, F. 2003, ApJ, 590, L99

Denissenkov, P. A. \& Hartwick, F. D. A. 2014, MNRAS (Letter), 437, L21

D'Ercole, et al. 2010, MNRAS, 407, 854

D'Ercole, et al. 2010, MNRAS, 423, 1521

Doherty, C. L., et al. 2014, MNRAS, 437, 195

Dotter, et al. 2010, ApJ, 708, 698

Forestini, M. \& Charbonnel, C. 1997, A\& A, 123, 241

Gratton, R. G., et al. 2010, A\&A, 517, A81

Karakas, A. \& Lattanzio, J. 2007, PASA 24, 103

Khalaj, P. \& Baumgardt,H. 2015, MNRAS, 492, 924

Gratton, R. G., Carretta, E., \& Bragaglia, A. 2012, ARAA, 20, 50 
Krause, M., Charbonnel, C., et al. 2012, A\&A (Letter), 546, L5

Krause, M., Charbonnel, C., et al. 2013, A\&AA, 552, A121

Krause, M., Charbonnel, C., Bastian, N., \& Diehl, R. 2016, A\&SA, in press, arXiv:1512.04256 (K15)

Kroupa, P., et al. 2013, in Planets, Stars and Stellar Systems (Springer Netherlands), 115

Lagarde, N., et al. 2012, A\&A, 543, A108

Larsen, S. S., et al. 2014, $A \mathscr{E} A, 565$, A98

Lind, K., et al. 2009, A\&A, 503, 545

Maeder, A. \& Meynet, G. 2006, A\&SA, 448, L37

Marino, A. F., et al. 2014, MNRAS, 437, 1609

Marino, A. F., et al. 2015, MNRAS, 450, 815

Martell, S. L., Molinski, J. P., Beers, T. C., \& Grebel, E. K. 2011, A\&AA, 354, A136

Milone, A. P., Marino, A. F., Piotto, G. P., et al. 2013, ApJ, 767, 120

Milone, A. P., Marino, A. F., Piotto, G. P., et al. 2015, ApJ, 808, 51

Piotto, G., et al. 2002, ApJ, 670, 39

Prantzos, N. \& Charbonnel, C. 2006, A\&SA, 458, 135

Prantzos, N., Charbonnel, C., \& Iliadis, C. 2007, A\&BA, 470, 179

Renzini, A., et al. 2015, MNRAS, 454, 4197

Sbordone, L., Salaris, M., Weiss, A., \& Cassisi, S. 2011, A\&BA, 534, A9

Schaerer, D. \& Charbonnel, C. 2011, MNRAS, 413, 2297

Sills, A. \& Glebbeek, E. 2010, MNRAS 407, 277

Simmerer, J., et al. 2013, ApJ (Letters), 764, L7

Smith, G. H. \& Norris, J. 1982, ApJ, 254, 594

Ventura, P., et al. 2001, ApJ (Letter), 550, L65

Ventura, P., et al. 2013, MNRAS, 431, 3642 